\title{
eJRIEPS
}

Ejournal de la recherche sur l'intervention en éducation physique et sport

Hors-série $N^{\circ} 1 \mid 2015$

Quelques aspects théoriques de la didactique des sports collectifs. Préparation aux concours de recrutement

\section{Débat d'idées, langage, interactions discursives et apprentissage des sports collectifs}

Jean-Francis Gréhaigne, Nathalie Wallian et Fabienne Brière-Guenoun

\section{OpenEdition}

\section{Journals}

Édition électronique

URL : http://journals.openedition.org/ejrieps/1293

DOI : 10.4000 /ejrieps. 1293

ISSN : 2105-0821

Éditeur

ELLIADD

Référence électronique

Jean-Francis Gréhaigne, Nathalie Wallian et Fabienne Brière-Guenoun, « Débat d'idées, langage, interactions discursives et apprentissage des sports collectifs », eJRIEPS [En ligne], Hors-série $N^{\circ} 1$ । 2015, mis en ligne le 01 décembre 2015, consulté le 03 octobre 2019. URL : http:// journals.openedition.org/ejrieps/1293; DOI : 10.4000/ejrieps.1293

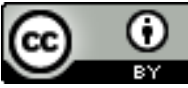

La revue eJRIEPS est mise à disposition selon les termes de la Creative Commons Attribution 4.0 International License. 
eJRIEPS Hors série n¹ Décembre 2015

Débat d'idées, langage, interactions discursives et apprentissage des sports collectifs

\author{
Jean-Francis Gréhaigne ${ }^{*}$, Nathalie Wallian ${ }^{* *}$ \& Fabienne Brière-Guenoun ${ }^{* * *}$ \\ * Professeur des Universités retraité, Université Bourgogne Franche-Comté \\ **Professeure de Sciences de l'Education, Université de la Réunion \\ ***MCF - HDR, Université Paris-Est Créteil
}

Dans les trente dernières années, les recherches dans différentes disciplines visant à construire des apprentissages ont contribué à faire de la question du débat, de l'échange d'idées pour construire des apprentissages, une question importante dans les pratiques d'enseignement (Brousseau, 1986 ; Perret-Clermont, 1979 ; Schubauer-Leoni, 1997). Plus précisément, en sciences expérimentales est apparu un mode didactique appelé « débat scientifique dans la classe » (Joshua \& Dupin, 1993). Néanmoins, il faut noter immédiatement que l'examen des premiers temps où les pratiques de débat se sont installées dans une classe a montré à l'évidence que l'idée selon laquelle il suffirait de mettre les élèves ensemble pour qu'ils dialoguent était plutôt fausse (Sensevy, 1998). Pour qu'il y ait un véritable débat scientifique, il faut que le professeur puisse simuler dans sa classe une micro-société scientifique afin que les connaissances soient des moyens utiles pour poser de bonnes questions et résoudre les problèmes (Brousseau, ibid.). Un débat en classe est une organisation pédagogique qui permet une discussion, autant que possible constructive sur un problème précis et à laquelle prennent part des élèves ayant des avis, réflexions ou expériences différentes sur le problème considéré. Le débat est un mode didactique qui considère la classe en tant que communauté (enseignant et élèves), communauté dans laquelle toute action est nécessairement conjointe (Sensevy 2007).

En éducation physique et sportive (EPS), il ne saurait y avoir de débat scientifique stricto sensus au gymnase ou au stade, du fait de la difficulté d'identifier et de bien cerner les concepts, les connaissances et les compétences à l'œuvre car ils relèvent d'une grande diversité de champs. Aussi, nous avons préféré parler de débats d'idées (Gréhaigne \& Godbout, 1998) même si cette qualification peut paraître vaste et trop générale. En effet, il nous a fallu tenir compte que la dénomination "débat scientifique " dans les autres disciplines, reste aussi problématique. En effet, dans une perspective de didactique disciplinaire, le débat ne joue pas tout à fait le même rôle dans toutes les matières. Par exemple, la part accordée à la confrontation des représentations ou à la résolution de 


\section{eJRIEPS Hors série n¹ Décembre 2015}

problèmes, sera différente suivant qu'on fait des mathématiques ou des Sciences de la Vie. Cependant, il existe des points communs. Rapidement, il faut faire un bilan des connaissances qui préexistent au débat entre élèves car les réponses de ceux-ci lors de la discussion seront filtrées par ces connaissances préalables. Ce débat où l'enseignant connaît les réponses est donc différent de ce qui se passe dans une communauté des chercheurs qui échangent sur des problèmes dont aucun ne connaît vraiment la solution. À l'école, cet aspect suffit à différencier cette forme de débat de toutes les autres formes possibles de débats avec en plus en EPS une filiation fondamentale à une pratique sociale de référence (Martinand, 1981) connue plutôt qu'à un corps de connaissances. Évidemment, les discussions et les débats entre élèves ou entre élèves et l'enseignant impliquent une verbalisation partagée. Caverni (1988) a montré que la verbalisation pouvait être une source d'informations et d'influence sur les processus cognitifs. Considérant le moment du débat par rapport à l'exécution des tâches, il a distingué trois types de verbalisation : la verbalisation avant (en tenant compte ce qui sera ou devrait être fait), la verbalisation simultanée (en considérant ce qui est fait), et la verbalisation consécutive (compte tenu de ce qui a été fait).

Dans le débat d'idées, il s'agit de proposer des séquences orales où la parole est vécue comme une interaction en vue de produire des effets cognitifs. Cela met en scène des joueurs dans une situation qui vise la co-construction de savoirs. En bref, un contexte didactique où les apprenants ne se limitent pas à un rôle passif face à un maître ou un entraîneur détenteur du savoir (cf. Rabatel, 2004).

\section{Quelques données sur ces débats d'idées}

Concernant ce débat d'idées en EPS, Chang (2006), Deriaz, Poussin, \& Gréhaigne (1998), Dietsch, (2014 ; 2015), Gréhaigne \& Deriaz, (2007), Gréhaigne \& Godbout, (1998), Poussin \& Cotting (2011), Zerai, (2011), Zerai, Rezig \& Zhibi, (2008) ont montré l'intérêt d'une prise de distance sur l'action grâce à la construction de "règles d'actions ", elle-même permise par une autre perspective et organisation de l'enseignement. Cette dernière articule des temps de jeu, des moments d'observation d'une séquence de jeu et des temps de débat reposant sur l'analyse d'une séquence de jeu par un groupe d'élèves (généralement l'équipe qui vient de jouer pour les sports collectifs). Une critique souvent entendu à ce type d'approche consiste à affirmer que cela favorise encore les meilleurs, les plus habiles en termes de langage. Mais, selon Poggi, Musard et Wallian (2007), les modalités d'utilisation du débat d'idées sont corrélées au niveau scolaire et à l'origine 


\section{eJRIEPS Hors série n¹ Décembre 2015}

sociale de milieux défavorisé et favorisé sans que l'on puisse dire quel est l'usage le plus profitable en termes d'apprentissages. Le débat d'idées échappe ainsi en partie au poids de la variable socioculturelle, ce qui plaide en faveur de son utilisation en milieu difficile. Nous allons maintenant envisager quelques points clés par lesquels il nous semble nécessaire de passer pour réussir.

- Le changement de contrat didactique entre le professeur et la classe car il faudra en installer un nouveau. Le contrat didactique est le contrat, souvent implicite, qui dicte les rôles, les droits et devoirs des acteurs de la classe, professeur et élèves, sur le plan didactique.

- La dévolution du problème aux élèves. II s'agira d'une activité dont le seul but sera de faire comprendre aux élèves comment ils peuvent eux-mêmes trouver des réponses aux problèmes posés.

- Un retour au jeu car une fois la dévolution d'une certaine responsabilité initiée, il faut du temps pour que les élèves puissent tester leur décision avec succès ou non.

- Une alternance de ce que nous avons désigné comme des activités "de débat 》 qui introduisent des connaissances ou des pratiques nouvelles et des activités " ordinaires » qui renvoient à la situation d'opposition.

En résumé, conduire une approche constructiviste et cognitiviste dans une leçon de sport collectif en EPS, c'est faire en sorte que les connaissances et les compétences motrices du programme ne s'introduisent plus dans la classe à partir des seules propositions qui viennent du maître, mais plutôt à partir d'énoncés problématiques et des données que les élèves apprennent à formuler, à soutenir et à expérimenter ensemble. En ce sens, nous pensons que les débats entre les élèves que le professeur organise et structure pendant le cours ont alors pour objectif, non de faire tout découvrir, mais de confronter et de rapprocher les élèves du sens et de l'utilité de ces connaissances et de ces compétences En ce sens, ce texte vise une production et une intériorisation des savoirs. Cela signifie non seulement que l'élève comprend ce qui est essentiel, mais en plus que cela le transforme : il voit le jeu autrement, il se forge une représentation suffisamment pertinente de ce que représente ces rapports d'opposition pour en faire un outil personnel dont il va pouvoir se servir ultérieurement. Ces débats, qui sont par nécessité souvent peu ou pas argumentés en tout début du cycle, tendent à devenir de plus en plus structurés et pertinents dans l'avancée des cours tout en préservant le sens en modifiant peu à peu les conceptions / représentations. En effet, les arguments que les élèves apprennent alors à utiliser pour comprendre, se convaincre eux-mêmes et pour convaincre leurs pairs 


\section{eJRIEPS Hors série n¹ Décembre 2015}

deviennent ainsi de plus en plus spontanément ceux du raisonnement. II est clair qu'un enseignant ouvrira d'autant plus facilement un vrai débat en classe qu'il sera assuré que, lors de ses interventions son rôle consistera principalement à mettre de l'ordre lors des interventions et à compléter ce qui sera apparu dans le débat, plutôt qu'à devoir lutter contre des représentations conjoncturelles auxquelles certains élèves ne voudraient pas renoncer («le foot à 11, c'est le vrai foot... »). Dans l'élaboration des actions en projet, cela implique pour le professeur d'être actif et attentif aux processus en cours, de ne pas laisser voguer la parole, voire parfois de formuler des consignes et d'articuler des règles de fonctionnement en posant de réelles exigences visant à l'apprentissage. Le rôle des connaissances disponibles est fondamental et les expériences de Zerai (2011) soulignent bien ce fait car des jeunes filles Tunisiennes qui débutaient en handball restèrent coi lors du premier débat d'idées proposé. II faudra plusieurs leçons pour que l'expérience aidant il se passe quelque chose et que les actions en projet émergent. Ainsi, il semble bien que le facteur le plus influent en ce qui concerne la mobilisation de procédures s'avère être la possibilité pour l'élève de mobiliser des connaissances préalables relatives au domaine concerné.

Beaucoup de théories d'actions (Lesnes, 1977) en vogue, concernant les apprentissages moteurs, rabâchent en boucle que quand on a fini de répéter, on recommence pour viser une soi-disant perfection du geste. Cela peut éventuellement se concevoir dans les activités morphocinétiques de haut niveau mais cela constitue, bien souvent, un discours de manager ou de coach pour décerveler et assujettir le pratiquant. Dans les activités comme les sports collectifs où la durée de l'affrontement est centrale, les choses diffèrent. Ici, deux théories s'affrontent : d'un côté ceux qui défendent quand même le geste parfait, libérateur de l'esprit et de l'initiative en vue d'une application au jeu; et de l'autre ceux pour qui une personne représente un tout unique avec ses états d'équilibres et de déséquilibres particuliers, avec un fond culturel commun certes mais aussi une originalité non réductible. Dans ce cas, la technique corporelle est un objet culturel que les individus selon leur univers de référence et leur système de représentation spécifique s'approprient en le transformant. Chaque pratiquant redéfinit cet objet au regard de ce qu'il connaît, de ce qu'il croit connaître et comprendre et de ce qu'il a envie de faire (Bride, 2015). Nous visons donc, au travers du débat d'idées, une auto-socio-construction des compétences motrices et connaissances tactiques avec une approche que nous qualifierons de " sociosémio-constructiviste ». 


\section{eJRIEPS Hors série n¹ Décembre 2015}

Ainsi, les élèves, acteur de leur propre formation, développent une activité de mise en relation consistant à comparer le but mis en perspective avec le résultat atteint, puis à analyser les raisons de l'échec et / ou de la réussite permettant ainsi de faire évoluer la planification, les actions en projet et les ressources motrices utilisées. Dans ce cas-là, on peut considérer que les joueurs progressent, quand ils apportent quelques réponses positives dans des situations contraignantes si celles-ci sont nouvelles pour eux. Des objectifs, plus lointains, dans un cycle de travail pourraient viser, par exemple, à diminuer le nombre de pertes de balles dans une situation de confrontation identique et à augmenter le nombre d'actions de marque, à réaliser la même performance avec une augmentation des contraintes (densité des joueurs, espace plus restreint, vitesse plus importante, buts plus petits...) ou à diminuer l'écart avec une performance réalisée dans des conditions plus favorables.

Cela permet aux joueurs de travailler sur des objectifs directement mesurables. En définissant notamment très précisément les critères de réussite, ils peuvent ainsi mieux concrétiser les modifications de leurs comportements et leurs acquis. Ces données objectives facilitent pour les joueurs et l'équipe la gestion des objectifs à atteindre. II nous semble que ce type de conception de la formation, sans négliger les aspects gestuels qui devraient, quand même, être travaillés le plus souvent en situation d'opposition, permettrait au joueur la construction et l'appropriation à côté des habiletés motrices nécessaires au jeu comme par exemple des indices pertinents à prendre en compte, des règles d'action susceptibles d'orienter l'activité sans la limiter à la seule imitation de l'expert, pris ... comme modèle absolu ou encore des règles de l'organisation du jeu.

Autrement dit, à côté de l'action qui se satisfait de la notion de réussite (le but est atteint ou non), nous proposons, comme élément indispensable à la formation d'un joueur, la prise de conscience des conditions de la réussite qui, seule, permet l'interprétation et l'explication de l'action. Nous défendons la thèse que cette centration sur les mécanismes qui ont produit l'action permet d'engendrer, d'une part, de nouvelles actions dans la mesure où l'on a compris ce que l'on a fait ouvrant ainsi, de nouvelles possibilités. D'autre part, en tant que modèle explicatif, cette prise de conscience peut être le point de départ d'une série d'autres conceptualisations avec comme corollaire un champ de généralisation beaucoup plus large. Dans ce cas, on enrichit les images opératives (Ochanine, 1978), le cadre de référence des perceptions et de l'analyse du jeu en faisant évoluer les éléments pris en compte et, ainsi, on modifie les décisions prises... 


\section{eJRIEPS Hors série n¹ Décembre 2015}

Les temps de confrontation et de réflexion permettent aussi aux élèves d'acquérir, aussi, une démarche qui les rend plus conscients de leur prestation. Deriaz et Poussin (2001) insistent aussi sur le fait que l'on devrait retrouver ces temps tout au long des différentes phases du processus d'apprentissage. L'ensemble de ces attitudes face à une production motrice doivent les conduire à mieux planifier leur jeu avant ou pendant l'action (Lafont \& Winykamen, 1999). La mise en évidence des liens entre les différents indices utilisés pour l'observation doit soutenir la réflexion des élèves sur leur propre pratique. Nous sommes bien dans la perspective de l'appropriation de connaissances et le développement de compétences variées en EPS afin de permettre à chacun de transformer sa pratique de façon durable en la rendant plus raisonnée.

\section{Débats d'idées et sport collectif}

A la différence d'une association ou d'un club, l'école obligatoire est un lieu où l'on accueille tous les élèves, avec des motivations et des niveaux très différents. Les instructions officielles sur l'enseignement de l'EPS recommandent l'apprentissage de connaissances et de compétences avec la collaboration active des élèves. Dans l'enseignement secondaire, avec 750 heures d'enseignement et de nombreuses familles d'APSA, il est possible de consacrer environ 200 heures au maximum en sport collectif. Quels peuvent être les objectifs que l'on peut raisonnablement atteindre au terme de ces cycles d'apprentissage ? Comment développer les tactiques des élèves en situation d'affrontement en sports collectifs ?

2. 1. Une démarche socio-sémio-constructiviste chez les élèves

Dans cette "situation de pratique scolaire " (Le Bas, 2008) des élèves jouent à un sport collectif arbitré par un de leur camarade. Le professeur et les observateurs regardent le jeu et le comportement des joueurs. Chacun des observateurs s'appuie sur son cadre de référence personnel, celui-ci pouvant néanmoins être négocié et en partie partagé (Gréhaigne, Godbout, \& Bouthier, 2001). Dans ces conditions, le rôle de l'enseignant consiste à attirer l'attention du joueur sur sa façon d'agir, sur les moyens employés pour gagner la partie. A partir d'indicateurs qualitatifs ou quantitatifs, il favorise la mise en rapport des indicateurs avec des données objectives collectées par les observateurs et avec le résultat de l'action. 


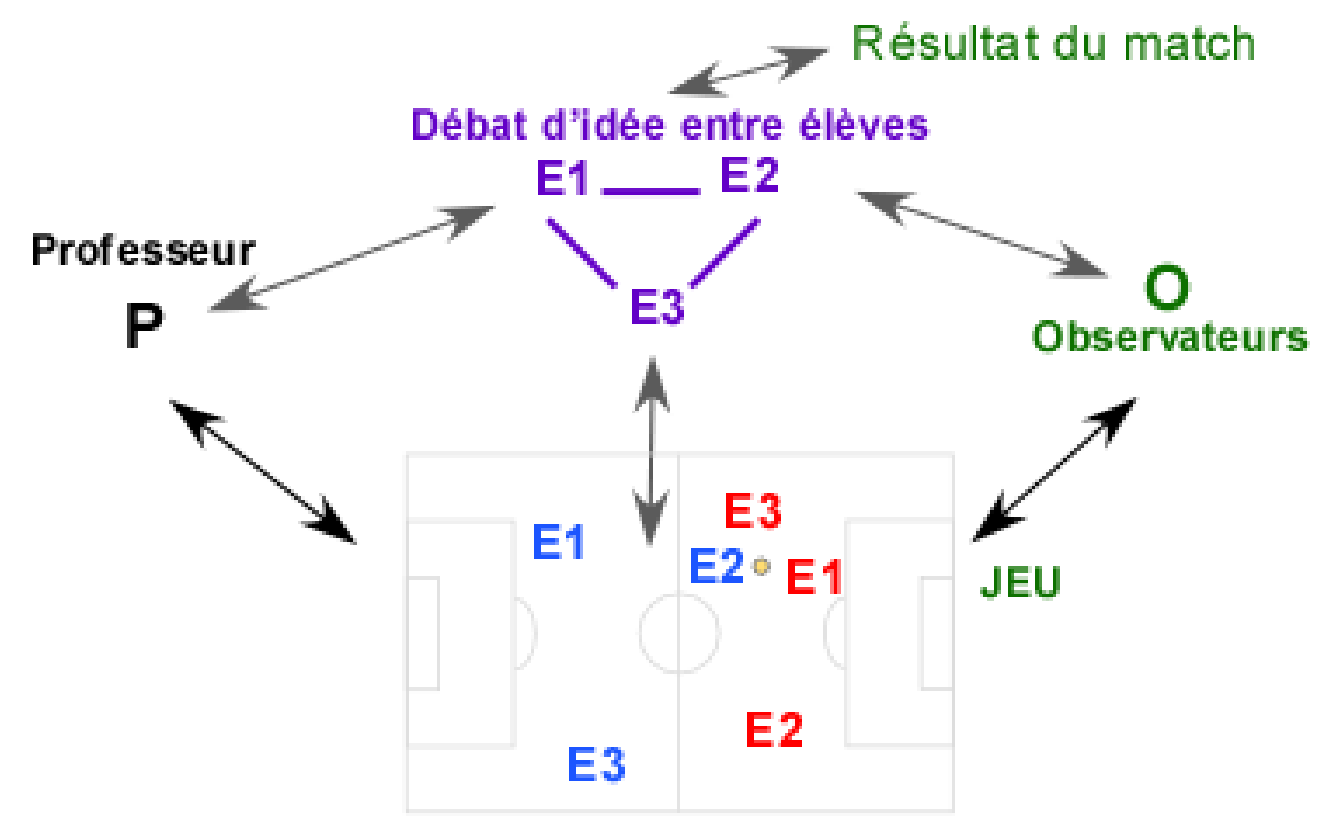

Figure 1. Une situation d'enseignement avec débat d'idées et observations.

Cette situation d'enseignement / apprentissage doit être construite pour que s'engage une activité scolaire, c'est-à-dire pour créer une rupture avec les usages habituels et les compétences déjà présentes. La référence à la théorie des situations-problèmes est clairement revendiquée mais la résolution de problème n'apparaît pas comme une fin en soi mais comme la recherche d'une réponse adaptée dans une situation où plusieurs sont possibles (Le Bas, 2008).

2. 2. Un cycle, une unité temporelle d'apprentissage Il faut du temps pour apprendre. En effet, tout apprentissage prend du temps et à l'école aussi il faut donner du temps au temps si l'on veut que les élèves apprennent quelque chose. Alors donnons du temps aux élèves avec des cycles suffisamment longs ou rapprochés pour leur permettre de stabiliser les nouveaux apprentissages. Des cycles de 10 à 12 leçons de deux heures semblent un minimum pour passer de réponses nouvelles à des apprentissages stabilisés pour les élèves. 


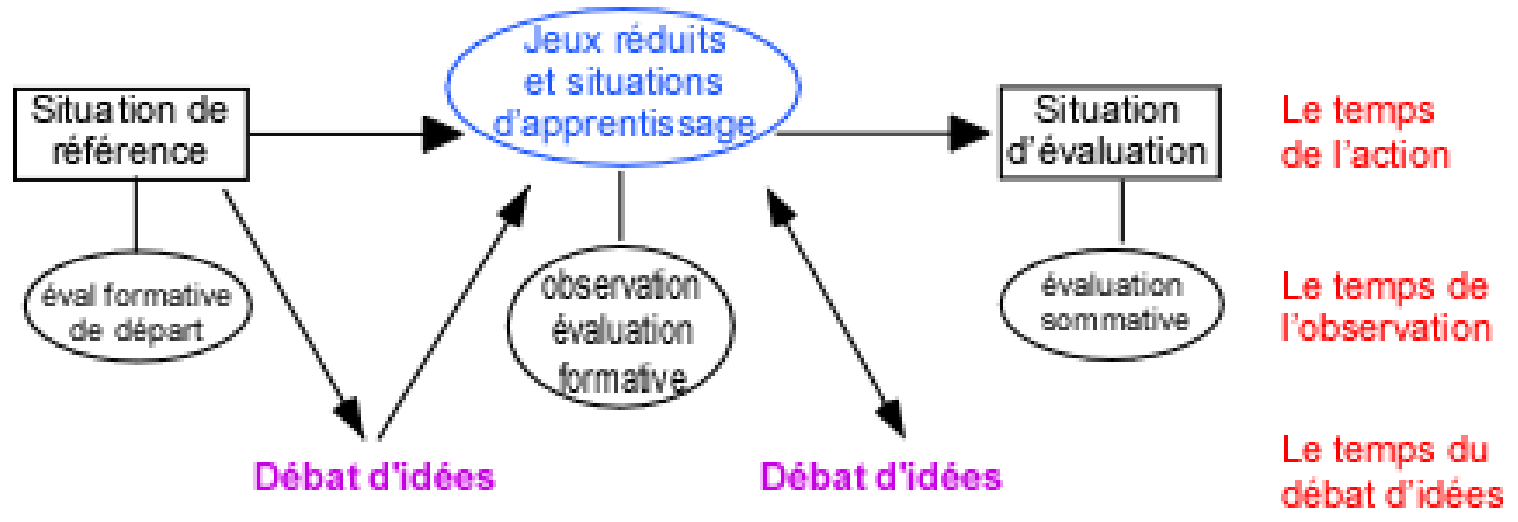

Figure 2. Modèle didactique pour l'enseignement des sports collectifs à l'école.

L'approche tactique des jeux sportifs collectifs propose trois temps différents qui sont utiles aussi bien dans les "situations de référence ", dans les " jeux réduits et situations d'apprentissage » que dans les « situations d'évaluation » :

- Les temps d'action où les élèves sont en activité motrice. C'est la situation classique où des élèves, face à une tâche proposée par l'enseignant, jouent et essaient de résoudre le problème posé.

- Les temps d'observation où les élèves qui ne participent pas aux activités motrices, relèvent des informations en fonction des critères chiffrés définis précisément. Ces données serviront soit pour l'évaluation formative, soit pour l'évaluation sommative.

- Les temps de "débat d'idées" (Gréhaigne \& Godbout, 1998 ; Deriaz, Poussin, \& Gréhaigne, 1998) sont des situations dans lesquelles les élèves s'expriment et échangent à propos du jeu..

Ce processus est rendu possible par un travail collectif d'analyse qui permet l'évaluation et la validation des énoncés de connaissances et de savoirs d'action construits au cours de la pratique sur des critères d'efficacité et de pertinence au regard de l'action. 


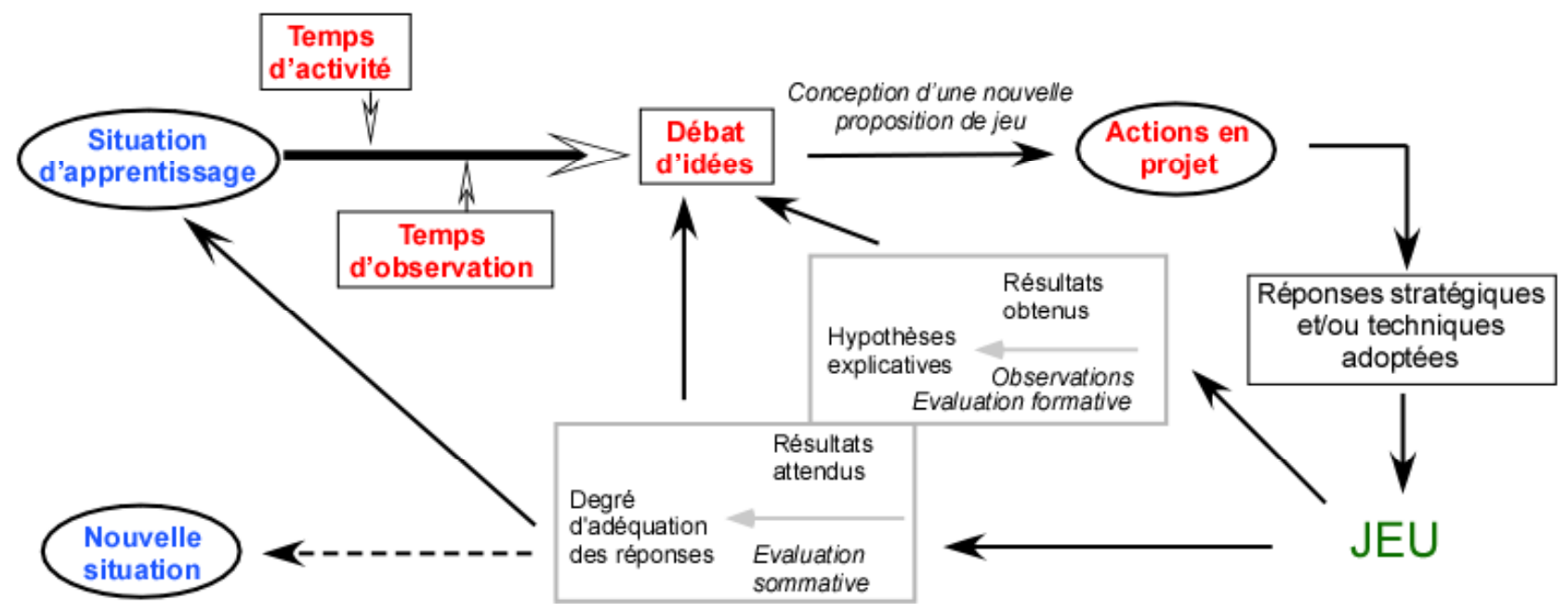

Figure 3. Diverses facettes de l'utilisation de l'évaluation formative dans un débat.

La figure 3 illustre aussi l'autoévaluation possible des stratégies et tactiques utilisées réellement ainsi que la construction collective et individuelle du sens de l'action. Enfin, pour l'enseignant, il est nécessaire d'identifier les pratiques effectives des élèves et non pas ce qu'ils ne savent pas faire en relation avec, parfois, une évolution souhaitable de la situation.

2. 3. Une approche tactique de l'enseignement des sports collectifs

Le débat d'idées constitue toujours une pièce centrale d'une conception socio-sémioconstructiviste de l'apprentissage des sports collectifs. Avec un retour d'informations chiffrées, le débat consiste après une séquence jouée à effectif réduit, en une discussion destinée à faire évoluer ou non les actions en projet de l'équipe en revenant sur le score, la stratégie prévue et en analysant la tactique appliquée. Un thème qui revient souvent consiste, lors de l'établissement d'actions en projet sous forme "d'une feuille de route ", à avoir un projet de jeu, une stratégie. La mise en place de cette feuille de route provoque bien souvent la proposition de circulations fantaisistes du ballon et des joueurs. Le fait de parler d'actions en projet souligne bien l'interaction sujet / environnement dans toute prise de décision ou tout projet d'action en relation avec la nécessité de proposer une « feuille de route » réaliste. 
Vers la ré-utilisation

des connaissances et

des compétences apprises

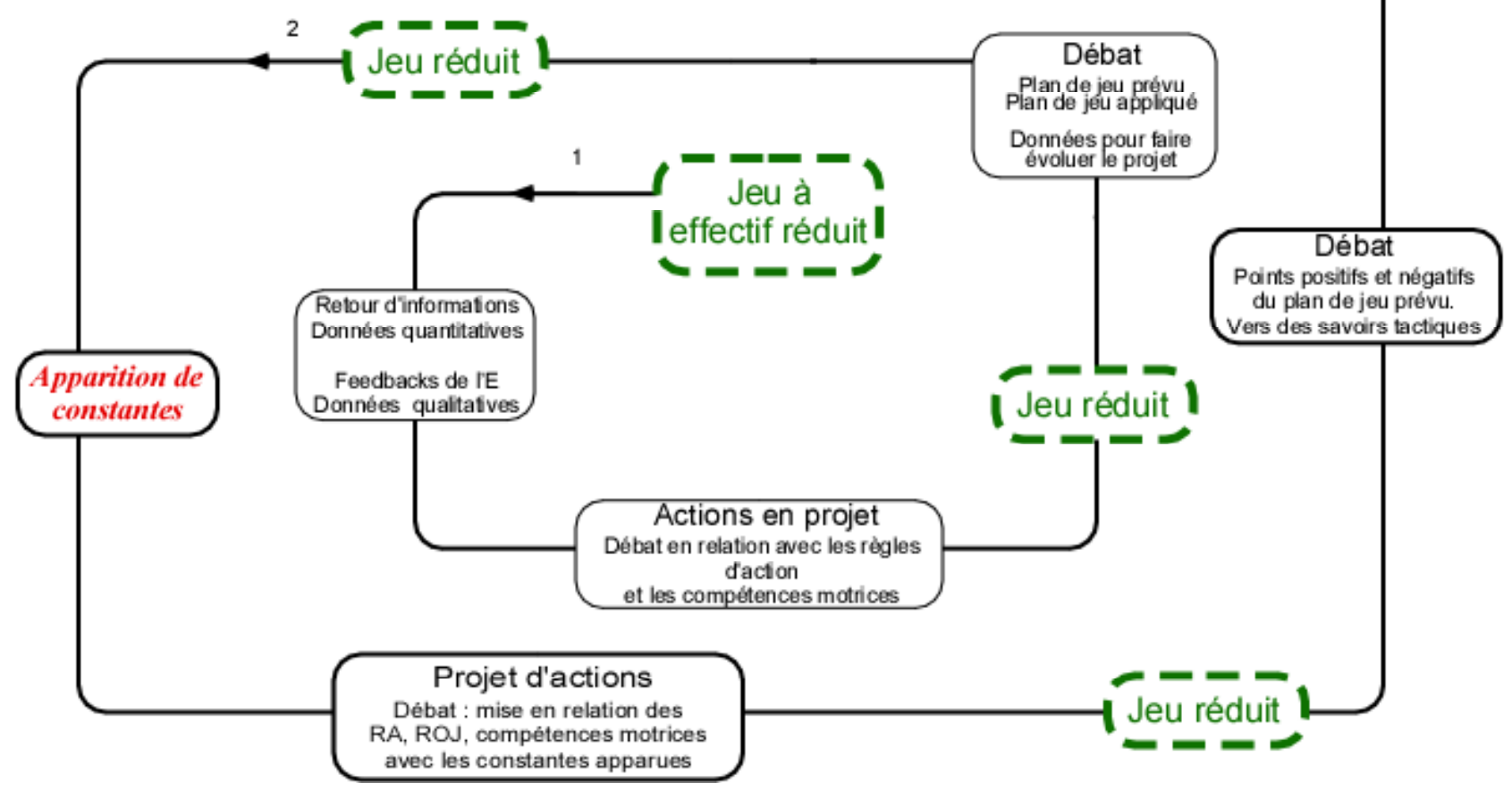

Figure 4. Modélisation de l'approche tactique de l'enseignement des sports collectifs.

La gestion des débats dans ce type de démarche présente des caractéristiques bien précises. Le débat d'idées ne doit pas durer plus de deux à trois minutes. Si le professeur désire faire un apport dans cet échange, cette intervention doit être brève et concise pour éviter de parachuter des solutions toutes faites. II ne faut pas changer la situation de jeu trop souvent mais se servir des contraintes et des consignes pour la faire évoluer dans le sens d'une facilitation ou d'une augmentation de la difficulté de telle façon que les élèves puissent développer une authentique activité de transformation par la recherche active de réponses judicieuses. Dans cette perspective, les élèves peuvent proposer des évolutions de la situation et l'enseignant devient alors personne-ressource pour des joueurs qui analysent les moyens dont ils disposent actuellement et qui possèdent un projet de transformation.

Cette conception de l'apprentissage au sein d'une activité que nous qualifierons de sociosémio-constructiviste permet de déployer chez les élèves une activité de problématisation. II s'agit ici d'établir une relation dialectique entre le connu et l'inconnu en se questionnant à propos d'une action où il est raisonnable de suspecter un problème. En sport collectif, la problématisation s'oriente vers la recherche d'une réponse en actes aux problèmes qui vont surgir de l'interaction sujets / rapport d'opposition. Pour le joueur, cela nous renvoie 


\section{eJRIEPS Hors série n¹ Décembre 2015}

bien à une démarche d'auto-socio-construction soulignant par-là que l'acte d'apprendre est un acte singulier, individuel (on n'apprend rien au joueur, c'est toujours lui qui apprend) mais que cet apprentissage se conduit dans un cadre socialisé.

\section{Débat d'idées et apprentissage en sport collectif}

Tout au long du déroulement d'un échange communicatif, les différents participants, que l'on nommera "des interactants ", exercent les uns sur les autres une influence qui coordonne, harmonise en permanence leurs comportements respectifs (Wallian \& Chang, 2007). Autrement dit, un dialogue se construit dans l'échange. En effet, Jacques (1979) considère que "pour qu'un rapport de communication puisse s'instaurer, il faut cette différence initiale, de nature et de quantité d'informations, qui seule explique que l'un commence à écouter et que l'autre se fasse entendre » (p. 195). Selon la direction de la discussion, ces temps de débat d'idées peuvent poursuivre des objectifs différents possédant certaines similitudes avec des situations proposées par Brousseau (1998) dans sa théorie des situations. En effet, il peut présenter les caractéristiques de :

- situation de formulation, dans laquelle le savoir a une fonction de justification ;

- situation de validation, dans laquelle le savoir a une fonction de solution ;

- processus de généralisation, dans lequel le savoir a une fonction de référence.

De toute façon, en fonction du degré d'adéquation des réponses par rapport à l'effet recherché, il y a soit modification des moyens mis en œuvre pour réduire les écarts avec les effets attendus, soit stabilisation des réponses, soit complexification de la situation en vue d'amener l'élève à un niveau de pratique supérieur.

Avec le débat d'idées, nous entrevoyons deux axes d'approfondissement. Le premier concerne le choix des modalités de l'échange. En effet, le moment et la place des acteurs (enseignant et élèves) dans ce débat constituent un point capital.

- Quel est le meilleur moment pour ce débat afin de ne pas casser le processus didactique?

- Le débat est-il sous le contrôle unique de l'enseignant (c'est-à-dire que c'est lui qui pose toutes les questions comme c'est lui qui "réceptionne" les réponses des élèves) ?

- Le débat est-il "interactif" ? L'enseignant assume principalement un rôle de médiateur dans les débats entre élèves.

- Le débat d'idées est-il engagé, mené, alimenté par les seules interactions entre les élèves, l'enseignant constituant seulement une personne ressource? 
eJRIEPS Hors série n¹ Décembre 2015

Le second se rapporte au type de questionnement qui oriente et dirige le débat quand l'enseignant décide d'intervenir.

- Le questionnement doit-il être ouvert (c'est-à-dire que le questionnement pose un problème aux élèves et que les relances sont référées aux réponses précédentes) ?

- Le questionnement doit-il être fermé (le questionnement progresse par réorientation et/ou refus des réponses "fausses" jusqu'à l'obtention de la réponse souhaitée) ?

- Le questionnement doit-il être "attentiste" (c'est-à-dire qu'il doit laisser se dérouler le débat jusqu'à ce qu'apparaisse la réponse attendue) ?

Ces trois derniers types de questionnements représentent ainsi des structurations possibles du débat d'idées, qui peuvent prendre des formes variés, en fonction du type de classe, du profil pédagogique, des conceptions pédagogiques des enseignants et des stratégies d'apprentissage des élèves. II est bien évident que cette façon de travailler dépend en partie du type de classes et surtout de leur composition. Avec des classes difficiles de la périphérie urbaine, il faut d'abord penser à jouer en évitant si possible les conflits. Dans un second temps, il est souvent aisé d'introduire un débat d'idées dirigé qui permet aux élèves d'échanger sur des faits pratiques. La notion de tour de parole peut constituer alors un enjeu important de ce style de travail. Avec les autres types de classes, il est tout à fait possible d'utiliser le débat d'idées sous des formes variées.

Avec le recul, des règles de fonctionnement du débat d'idées ou de la verbalisation semblent se dégager. Les recherches de Darnis (2004) et Darnis, Lafont, \& Menaut (2007) montrent que pour être efficace et pour donner lieu à des progrès individuels, le fonctionnement d'un conflit socio-cognitif doit remplir d'autres conditions que l'opposition initiale et la coordination finale entre les partenaires. Les auteurs distinguent deux groupes de contraintes les unes liées aux capacités individuelles des protagonistes, les autres au déroulement de l'interaction. II faut souvent attendre le troisième tour de parole pour que le débat démarre vraiment de façon constructive. Nachon (2004) attribue deux grandes fonctions aux interlocutions langagières : une fonction de description/analyse et une fonction de communication. "Elles permettent l'échange entre les individus de même que la construction des verbalisations projectives par la mise en avant des perceptions issues des divers engagements dans l'action. Elle repose donc sur un principe de la plurifonctionnalité » (p. 110).

En ce qui concerne le mécanisme, dès que les élèves font une proposition, cette donnée est mise à la disposition du collectif. De la sorte, l'élève qui avance la proposition devient un proposant qui, la plupart du temps, amorce une piste de réflexion. Cette fonction n'est 


\section{eJRIEPS Hors série n¹ Décembre 2015}

pas anodine. Aussi, les données peuvent s'enchaîner jusqu'à ce qu'il y ait, à un moment précis, "une prise de décision par l'entité collective». Ensuite, lorsqu'une donnée est avancée par un proposant, (1) soit elle reste en suspend, en n'étant ni fausse et/ou inutile, ni vraie et/ou importante mais en ne faisant simplement pas l'objet de commentaires, (2) soit elle se constitue comme une piste pour le discours à venir, en permettant l'embrayage de séquences conversationnelles. L'ensemble de ces attitudes face à une production motrice doit conduire les élèves à mieux planifier leur jeu avant ou pendant l'action (Lafont \& Winykamen, 1999). La mise en évidence des liens entre les différents indicateurs utilisés pour l'observation doit soutenir la réflexion des élèves sur leur propre pratique. Nous sommes bien dans la perspective de l'appropriation de connaissances et le développement d'aptitudes variées en éducation physique et sportive afin de permettre à chacun de transformer de façon durable sa pratique en la rendant plus raisonnée.

Pour conclure ce paragraphe, nous pouvons dire que le recours à des situations de verbalisation, impliquant l'enseignant et les élèves, dépend en grande partie de l'option privilégiée par l'enseignant pour les apprentissages. Dans la perspective constructiviste des apprentissages défendue par l'école, nous avons comme hypothèse forte que l'élève apprend mieux après avoir identifié les mécanismes qui conduisent à la réussite. Cette conception s'oppose aux tenants d'une «éducation physique transpiratoire ». Pour Gréhaigne et Laroche (1994), I'EPS "consiste aussi dans une large mesure, en l'exploration du champ des connaissances, en rapprochement analogique ou contradictoire des différents éléments de la situation et en l'effort du sujet pour répondre de façon plus cohérente et plus économique aux systèmes de contraintes qui lui sont proposés (p. 14) ». Quels sont les éléments théoriques à notre disposition pour soutenir une telle affirmation?

\section{Pratique et théorie : quels apports aux débats ?}

Ainsi, en EPS, l'essentiel des apprentissages consiste à " apprendre à faire » en rapport avec des connaissances. A ce propos, nous avons déjà écrit sur les rapports compliqués entre pratique et théorie (Gréhaigne, 1992). De la simple application de théorie produite ailleurs sur la pratique à une extraction exclusive de connaissance issue de cette pratique, le champ d'investigation est large. Nous allons donc envisager, dans la suite de ce paragraphe quelques contributions conceptuelles à la pratique de l'éducation physique et des sports collectifs. 


\section{eJRIEPS Hors série n¹ Décembre 2015}

4. 1. Pratique et théorie : pour un interactionnisme socio-discursif

Les apports de l'interactionnisme socio-discursif en EPS et, en particulier, dans les situations d'opposition, consiste à conduire des travaux théoriques et empiriques qui soulignent le rôle fondamental de l'activité discursive dans le développement des individus. L'intrication systémique pensée/langage/action accorde ainsi une place pour dire l'expérience et penser l'action et ouvre des perspectives d'investigation au chercheur qui veut accéder à l'activité de l'élève de façon internalisée. Pour ce faire, il s'agira de mettre en relation des sources croisées de cette activité langagière du joueur en situation d'apprentissage aux fins de donner du volume aux données analysées. Interpréter une action consiste donc à choisir des significations plausibles en les inscrivant dans un univers projeté de possibles, selon des points de vue à croiser et dans un univers de références partagées avec autrui au sein de la communauté des pratiques. Parler d'interprétation(s) de l'action, c'est présupposer qu'une lecture directe du mouvement ne suffit pas pour que le(s) sens de l'action soi(en)t épuisé(s) puisqu'il(s) n'est(ne sont) pas directement établi(s) derrière les gestes-signes chargés de le(s) porter (Wallian, 2010 ; 2010 a).

L'enjeu est donc le suivant : comment un sujet co-construit-il avec ses pairs une activité signifiante en situation didactique pour s'en trouver modifié, apprendre voire partager un savoir d'expérience ? En accord avec Austin (1965), nous postulerons pour cela que tout échange requiert une posture d'altérité dans laquelle il s'agit d'accueillir la nouveauté et le point de vue d'autrui en acceptant de s'en trouver transformé. Le croisement des interprétations entre pairs revêt alors un intérêt majeur. Ce croisement crée, lors du débat, un conflit d'interprétations (Ricoeur, 1986), qui se co-construit dans l'interaction argumentative et/ou dans la négociation des rapports de places. Cela autorise l'émergence de savoirs d'actions à partager au sein d'une communauté de pratiques en cours d'élaboration (Lave \& Wenger, 1991 ; Sensevy, Mercier, Schubauer-Leoni, \& Leutenegger, 2007).

Ici, l'objet est bien sûr de réhabiliter le rôle de l'action comme expérience première mais aussi celui de l'interaction langagière comme condition même de réalisation et d'actualisation des savoirs dans et par l'expérience. L'action est ainsi définie comme une intervention sur le monde en fonction d'effets à produire sur autrui (Bronckart, 1996). Le joueur devient dans ce cas un acteur responsable formulant des actions en projet. Le langage porte et incarne la pensée en actes et son étude permet d'accéder à une part du monde et aux connaissances telles qu'apprises, utilisées et vécues par le pratiquant. 
eJRIEPS Hors série n¹ Décembre 2015

"Les verbalisations à propos de l'action sont certes des reconstructions qui s'inscrivent inévitablement dans ces processus en oeuvre. A ce titre, elles ne seront pas considérées que comme le témoignage complet, strictement fidèle et exclusif de la pratique de l'acteur qui les formule: la comparaison des pratiques déclarées/intentionnées/constatées n'est pas superposable et comporte une part d'irréductible. Elles seront, néanmoins, prises comme outil réflexif de mise à distance de l'action et de conflit des interprétations " (Wallian, 2010, p. 7).

4. 2. Pratique et théorie : le rôle des connaissances

En éducation physique et sportive, l'expérience nous a souvent montré qu'il ne faut jamais s'attendre à une "application " directe de savoirs théoriques issus de la recherche. En effet, l'étude approfondie de la pratique, mélange contrasté d'idées et d'actions complétées par l'expérience du joueur, constitue un objet de recherche difficile à cerner et à appréhender. En conséquence, la théorie est continuellement décriée, cependant Kurt Lewin ne disait-il pas : «ll n'y a rien de plus pratique qu'une bonne théorie»? Cependant, on peut dire qu'il subsistera toujours un écart entre la théorie et l'action. C'est d'ailleurs ce qui a inspiré les travaux de Schön (1994) sur la pratique réflexive car si d'une théorie, on pouvait déduire à coup sûr la bonne décision, il suffirait de former des praticiens applicationistes.

Quand on parle de "théorie », on se place d'un point de vue spéculatif. C'est celui de la pensée génératrice de considérations abstraites qui excluent les aléas. Si on se situe du côté de la "pratique ", on se place du point de vue de l'action, qui est génératrice de résultats concrets et qui incluent les aléas. Aussi, la théorie est généralement dévalorisée au profit de la pratique. Cela suppose que la théorie soit une spéculation impuissante coupée de la pratique dont on vante, par ailleurs, l'utilité et l'efficacité. Mais toute théorie n'est pas pure spéculation. Apparemment opposées, la pratique et la théorie sont en fait étroitement liées. II y a complémentarité entre la capacité qu'a le joueur d'agir concrètement sur lui-même et sur le jeu. Son aptitude à la pensée tactique est pour lui une source de connaissances sur les configurations du jeu. La théorie tire en effet de l'analyse de la réalité du jeu des règles d'action et des principes généraux. La pratique se nourrit de son dialogue avec la théorie pour mieux satisfaire l'efficacité.

Pourtant, face au système complexe que représente une rencontre de sport collectif, la tentation est souvent grande d'adopter une logique déductive et réductionniste : la simplification à valeur heuristique atteint pourtant, ici, ses limites. La première tâche consiste, souvent, à faire évoluer les questions vers une posture de chercheur, qui serait 


\section{eJRIEPS Hors série n¹ Décembre 2015}

la condition première de production de connaissances valides sur les phénomènes observés. De la théorie vers la pratique, certes la validité interne de la recherche décroît car la capacité à contrôler des facteurs expérimentaux au laboratoire fournit des informations de bonne valeur sur la variable isolée. Dans la pratique, la validité externe s'accroît fournissant ainsi des données utilisables immédiatement dans le jeu (Christina, 1987).

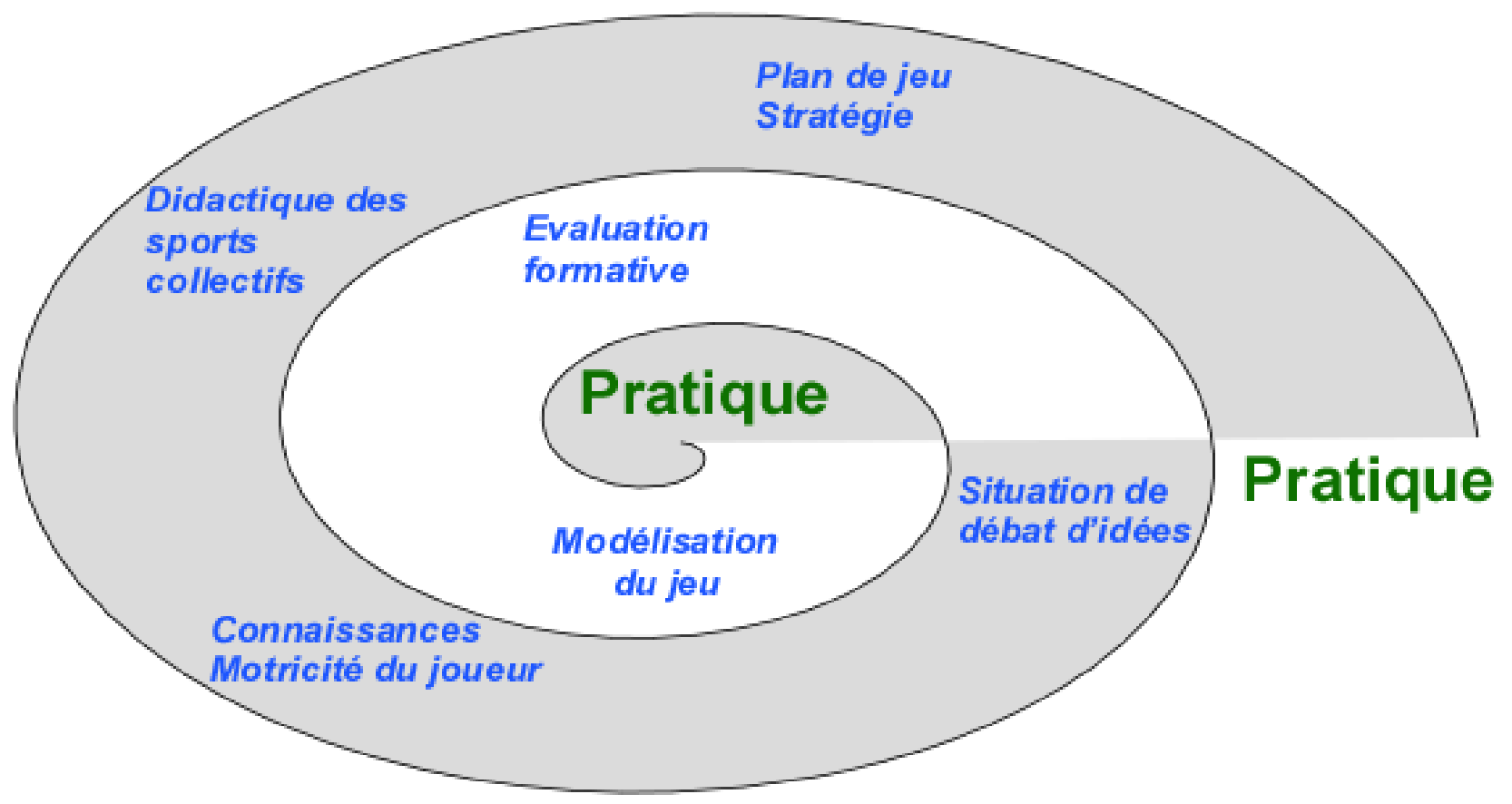

Figure 5. Incorporation de connaissances au cours de la pratique des jeux.

Par rapport à l'application de théories, la notion d'incorporation de connaissances semble bien convenir. Cela évite de se retrouver dans une logique déductive par rapport à des connaissances qui ont été produites dans d'autres champs et dans d'autres circonstances et que l'on peut rarement généraliser aux situations de la pratique. Donc, nous préférerons un modèle bâti selon un principe inductif.

Néanmoins, de la pratique au discours logiquement construit qui le décrit, il y a donc une accumulation d'indéterminations. II est pourtant relativement facile d'annuler quasiment toute cette indétermination. En partant du discours, il suffit de voir si l'événement prévu par le discours se trouve bien réalisé. C'est le passage de l'hypothèse au contrôle expérimental qui évalue la pertinence de l'analyse. Dans cette perspective, la connaissance apparaît dans un double mouvement, de la pratique à la modélisation et la modélisation à la pratique. Les connaissances particulières sont alors réduites à des données modulant les relations fonctionnelles existant entre un sujet connaissant et son environnement. C'est la pratique, le sens qu'elle prend chez le joueur, les 


\section{eJRIEPS Hors série n¹ Décembre 2015}

questionnements qu'elle suscite qui sont alors premiers. La théorie ou les connaissances savantes sont interrogées, en second, par cette démarche pratique. Ensuite, les deux étapes donnent lieu à des hypothèses testées dans les conditions réelles de jeu. Dans cette perspective, pratique et théorie sont intimement liées et fonctionnent non pas hiérarchiquement, mais en "va-et-vient " grâce aux interactions constantes. Les questions successivement posées s'emboîtent les unes aux autres et participent d'un affinement de la problématique. Extraire des connaissances de la pratique, les évaluer, les confronter à des modèles théoriques et retourner à la pratique nous paraît la voie la plus sûre pour minorer les dérives d'une théorisation qui serait trop prescriptive du jeu.

4. 3. Pratique et théorie : le rôle des modèles d'analyse du jeu

En sport collectif le recours, entre autres, aux processus cognitifs conscients dans la gestion de certains aspects de la confrontation et de l'apprentissage a amené une profonde modification de la conception du jeu. Cette conceptualisation s'effectue à partir du fonctionnement effectif de l'organisation du jeu. Cela mène également à une meilleure connaissance d'une partie du réel qui permet une gestion du jeu mieux appropriée. Elle tend à élargir son assise au-delà des divisions traditionnelles des systèmes de jeu formel en lignes, en postes, etc., pour intégrer et lui substituer des aspects plus dynamiques comme des trajets, des vitesses et des trajectoires de joueurs ainsi que du ballon. La conceptualisation est une forme d'activité imaginative qui permet de percevoir et d'interpréter les comportements des partenaires et des adversaires à partir des éléments caractéristiques principaux de l'image opérative : la finalisation, le laconisme et les déformations fonctionnelles (Ochanine, Quaas, \& Zaltzman, 1972).

Pour une meilleure efficacité, l'utilisation de théories ne saurait se concevoir aujourd'hui sans un recours à une présentation formalisée inspirée des modèles conceptuels. L'objectif principal d'un modèle conceptuel est de transmettre les principes fondamentaux et les fonctionnalités de base du système qu'il représente. Une modélisation conceptuelle adaptée doit proposer de nouvelles perspectives et un ensemble d'outils nouveaux pour promouvoir le progrès dans le jeu.

Nous présentons figure 6, un exemple de modélisation pour l'observation et l'analyse du jeu en mouvement en sports collectifs. Notre ambition, avec cette idée de modélisation, correspond à l'action de production de modèles. Un modèle peut être défini comme un système figuratif reproduisant en partie la réalité sous forme schématisée la rendant ainsi plus compréhensible. La réalité n'étant ni limpide ni directement accessible, il faut souvent la décoder, la simplifier en ne conservant que les éléments et les relations qui semblent 


\section{eJRIEPS Hors série n¹ Décembre 2015}

pertinents pour le sujet traité. Un modèle est une construction, une structure que l'on peut utiliser comme référence, une image analogique qui permet de matérialiser une idée, un concept, une action qui sont rendus ainsi plus directement assimilables utilisables (Gréhaigne, Marle, \& Zerai, 2013, p. 06).

\begin{tabular}{|c|c|c|}
\hline $\begin{array}{l}\text { Avoir en tête pour } \\
\text { décider }\end{array}$ & $\begin{array}{l}\text { Dynamique organisation du jeu } \\
\text { (Les flèches indiquent les évolutions } \\
\text { potentielles dans la séquence de jeu) }\end{array}$ & Éléments observables \\
\hline $\begin{array}{l}\text { Références en arrière- } \\
\text { plan } \\
\text { - Matrices de jeu : } \\
\text { Offensives et défensives } \\
\text { - Configurations du jeu } \\
\text { prototypiques } \\
\text { - Ressources du joueur } \\
\text { Conscience immédiate } \\
\text { - Stratégie, plan de jeu } \\
\text { en fonction du réseau } \\
\text { de compétences } \\
\text { - Opportunités tactiques } \\
\text { - Score de la rencontre }\end{array}$ & Contraction $\begin{array}{l}\text { Logique } \mathrm{H} \text { contre } \mathrm{H} \\
\text { Logique de ligne } \\
\text { - adossee à la cible } \\
\text { - au front du ballon }\end{array} \mid$ Reculer & $\begin{array}{l}\text { Rapports d'opposition } \\
\text { - Avance / Retard } \\
\text { - Barrage / Poursuite } \\
\text { - Position sur le terrain } \\
\text { de l'espace de jeu } \\
\text { effectif (EJE) } \\
\text { - Interpénétration et } \\
\text { positions des espaces } \\
\text { des EJEO et EJED } \\
\text { Jeu en mouvement } \\
\text { Trajets, vitesses et } \\
\text { trajectoires de la balle et } \\
\text { des joueurs }\end{array}$ \\
\hline
\end{tabular}

Figure 6. Un exemple de modélisation pour l'observation et l'analyse du jeu en mouvement en sports collectifs.

Enfin, le modèle envisagé doit pouvoir décrire un certain ensemble de faits à travers une structure explicative générale que l'on doit confronter à la réalité pour voir si elle reste pertinente tout en fournissant une interprétation des rapports d'opposition facile à comprendre pour les joueurs.

Les transitions entre deux configurations du jeu sont toujours porteuses d'informations notables sur l'évolution du rapport de forces. Ici, la description d'états dynamiques permet de mieux comprendre, à un instant donné, comment les joueurs sont en mouvement et l'évolution probable du jeu. Ainsi, l'évolution du système peut alors se modéliser (Figure 6) en concevant une évolution discontinue dans le temps à partir de la mise en relation de 


\section{eJRIEPS Hors série n¹ Décembre 2015}

critères concernant les rapports d'opposition et le jeu en mouvement. La démarche repose essentiellement sur le concept d'activité et le rôle de l'expérience, référés à des possibilités anticipatrices et à des possibilités de réglages actifs du sujet (Piaget, 1974). Ici, action et réflexion sont définies comme étant en interrelation et se co-construisant dans l'interaction en fonction d'un contexte de jeu donné. L'exploitation de ce modèle lors d'un débat d'idées entre les joueurs avant ou après la situation jouée permet une confrontation argumentée des points de vue sur les situations vécues et sur l'orientation donnée à l'activité des joueurs. En ce sens, l'analyse devient un instrument d'action interpsychologique et social qui produit des dissonances (Clot, 2000) constituées d'accords et de désaccords. Ces dissonances, utilisées comme ressources et non comme jugement de valeur sur la pratique de pairs, permettent de saisir la dimension partagée de l'activité et de mieux appréhender les représentations personnelles. En ce sens, l'activité de discussion, à partir de la pratique, permet aux participants d'accéder à leur propre fonctionnement et à ceux de leurs pairs à partir de la pratique. L'analyse des actions déployées entraîne la mise à jour de données qui sont caractéristiques de ces actions et donne ainsi accès à la compréhension de l'activité des joueurs permettant de mieux saisir, aussi, les éléments qui font sens pour le sujet (Pastré, 1999).

\section{4. Pratique et théorie : la feuille de route ou les actions en projet}

La mise en mots du jeu est en elle-même une expérience nouvelle et parler du jeu contribue aussi à le construire. Cependant, si le joueur peut être performant au niveau des compétences motrices ou des activités intellectuelles et techniques recouvrant son activité en jeu, il peut éprouver de réelles difficultés à la mise en mots de cette activité. Définir les objets, leurs propriétés, leurs relations, leurs transformations, ainsi qu'à répondre aux exigences communicationnelles attendues dans le processus de validation demande beaucoup d'efforts. Lors du débat d'idées, le joueur est conduit à s'exprimer pour dire « où et comment ça se passe » de façon très générale, avec des échanges de paroles entre lui et ses partenaires. Les limites pour traiter ces événements sont donc le temps, la complexité ainsi que la cohérence entre les signaux et les réponses.

Une feuille de route est, donc un outil pédagogique qui acte les décisions prises lors du débat d'idées. Pour mieux la faire fonctionner, pendant un temps défini, deux observateurs recueillent des informations sur les deux équipes en présence. Lors du premier arrêt de jeu, les observateurs communiquent les informations à leur équipe sur leur propre production ainsi que sur l'organisation de l'équipe adverse. Le groupe 
eJRIEPS Hors série n¹ Décembre 2015

enregistre ces informations, les met en relation et prend des décisions pour la séquence à venir.

Par exemple, une équipe qui a des difficultés avec la circulation du ballon peut décider temporairement de tenter de récupérer la balle le plus près possible du but adverse à l'espace arrière de l'équipe attaquante et juste après la récupération afin de tirer le plus vite possible au but.

Cette décision doit devenir quantitativement observable afin de mesurer et d'objectiver la stratégie retenue. Ensuite les deux observateurs rentrent pour jouer et, de fait, deviennent les acteurs privilégiés des décisions prises. Deux nouveaux observateurs fournissent de nouvelles données et le processus se reproduit. Pour apprécier l'adéquation entre les décisions prises et le jeu produit pendant la séquence, il suffit de croiser le résultat de la rencontre, le nombre de buts marqués ou le nombre de balles conquises avec les observations sur les balles récupérées à l'avant de l'espace de jeu par les observateurs qui sont bien ainsi au centre du processus. La mise en relation de ces données fournit une évaluation fiable des actions en projet et de leurs résultats permettant de réalimenter les débats d'idées pour l'évolution ou la construction de nouvelles feuilles de route. L'enseignant peut aussi aider à la détermination de ce qui est à apprendre. II met ainsi en perspective des éléments à observer, il analyse et régule les feuilles de route voire il incite à relever des observables de plus en plus fins. Le professeur n'est donc pas absent du système enseignement / apprentissage car il possède, lui aussi, sa propre feuille de route. Dans tous les cas, celle-ci est à confronter avec celles des acteurs qui en définitive apportent leurs propres réponses.

Ici, l'évaluation formative aide l'élève à apprendre et à se développer. Elle participe donc à la régulation des apprentissages et du développement dans le sens d'un projet éducatif L'observation formative peut être instrumentée ou purement intuitive mais si on désire obtenir une observation de qualité rigoureuse et systématique, il faut s'en donner les moyens. Dans le but de rendre plus tangibles les implications pédagogiques de l'évaluation formative et de l'observation, il est possible de proposer un cadre opérationnel lié à la pédagogie visant la réussite. Avant de s'engager dans la situation d'apprentissage, les élèves doivent savoir ce que l'on attend d'eux et/ou avoir décidé ce qu'ils ont à faire. Les élèves et l'enseignant doivent savoir jusqu'à quel point la tâche est réussie ou non, ce qui est réussi et ce qui ne l'est pas; il faut donc recueillir l'information sur les performances constatées dans le prochain jeu. À priori, aucun type d'information n'est exclu, aucune modalité de saisie et de traitement de l'information ne doit être écartée. 


\section{eJRIEPS Hors série n¹ Décembre 2015}

L'accès des élèves à une terminologie spécifique, constitue un passage incontournable pour une communication claire et simple lors de temps consacrés aux débats d'idées. II est vrai que de nombreuses situations anecdotiques des sports collectifs et les caractéristiques individuelles des élèves ne permettent pas toujours de valider des règles d'actions strictes et rigides. A ce moment, les «logiques d'actions » qui déterminent les rapports logiques entre les différentes propositions à propos des actions sont issues de probabilités fortes constituant des indicateurs importants à réinvestir dans le cadre de débats d'idées élargis. Les travaux en technologie des APS sont là pour établir à partir de tendances fortes des «logiques d'actions" qui vont guider maintenant le processus d'apprentissages des élèves et des joueurs. Dans ce cas, le joueur se voit donc contraint d'évaluer, d'apprendre, d'intégrer, de s'approprier de nouveaux outils sous la pression de l'évolution du jeu. Les joueurs ou les élèves doivent donc prendre en compte les logiques d'évolution et se positionner de manière stratégique par rapport à celles-ci. Le travail d'analyse du jeu permet alors de co-construire des contenus répondant aux exigences d'un jeu pertinent, efficace et performant.

\section{Conclusion}

Dans le cadre d'un contrat didactique, organiser et gérer un véritable débat à visée d'enseignement / apprentissage en EPS consiste à organiser une activité de classe qui ne convoite pas uniquement des connaissances déclaratives. Ici, l'activité de débat apparaît comme un outil pour analyser la pratique et non comme un enjeu de savoir. Cela confirme que ce débat est à construire dans chaque discipline puisqu'il est fondé sur des fonctionnements spécifiques à cette discipline. Avec cette forme collaborative, l'identification et la distribution de tâches et la synchronisation des activités sont deux points clés de l'activité collective. II faut, d'une part, expliciter le rôle de chacun et, d'autre part, inscrire ces tâches dans une temporalité en vue de leur exécution. Dans cette collaboration, le point crucial est la construction d'un référentiel commun aux activités à mener à partir d'un constat sur la situation d'affrontement et de l'activité que chaque joueur peut y déployer. Dans la situation de jeu et dans le débat d'idées, l'activité collective a des exigences qui concernent la coordination des activités individuelles et leur organisation. On peut ajouter que si cette activité langagière permet de comprendre partiellement ce qui fait sens du point de vue du joueur, le chercheur va pouvoir exploiter ce matériau pour accéder à une meilleure connaissance de ces joueurs, de ses actions en projet, des indices sémiques qui font son horizon tel que construit. Cependant Rochex 
eJRIEPS Hors série n¹ Décembre 2015

(2010) nous met en garde sur le fait que « les différents protagonistes d'un processus de co-élaboration de sens au sein d'un dispositif d'intervention, de formation ou d'accompagnement, pourraient, voire devraient, être pensés et considérés, malgré leurs différences, non de statut social mais d'activité, comme protagonistes d'un processus de co-construction de connaissance me paraît non seulement infondée sur le plan épistémologique mais porteuse de ce que j'appellerais un risque de légitimisme inversé " (p. 118).

Néanmoins, en évitant cet écueil, un débat d'idées bien conduit autorise une mise à distance du joueur par rapport à lui-même et engage une prise de conscience ou une " conscientisation » (Deleplace, 1979) par un retour réflexif sur l'action. Cette prise de conscience peut être également attribuée à la mise à jour d'éléments routiniers dans des pratiques jugées efficaces par le joueur. Cette mise à distance et prise de conscience ont des effets avérés sur l'évolution des pratiques. A partir d'une certaine conscientisation du jeu, le joueur pourra l'orienter davantage car il est en train de construire ou possède un schéma abstrait rendant compte, de façon opératoire au niveau mental, de la logique d'actions correspondant à la situation évolutive particulière à laquelle se rapporte la configuration du jeu dans laquelle il est engagé. II peut le réaliser de façon opératoire, c'est-à-dire d'une façon qui lui permet d'agencer les éléments du jeu grâce à la possibilité d'anticipation mentale dans l'élaboration d'une suite de décisions. Tout cela se passe au fil de l'action car ce que le sport collectif semble souligner de plus en plus, c'est que le joueur doit être mentalement engagé, en même temps que physiquement et physiologiquement actif, s'il veut réussir dans les rapports d'opposition.

Enfin, les recherches en intervention sur le terrain de la didactique et la mise en place de nouvelles méthodes à propos des séquences didactiques constituent des éléments de base pour toute étude. Ceci implique l'analyse in vivo de la manière dont l'enseignant organise son activité quotidienne et l'identification des multiples ressources, connaissances ou compétences qu'il met effectivement en œuvre dans ce cadre. Cela souligne aussi, la nécessité de procéder à des recherches à propos du travail des élèves, visant à mettre en évidence des constantes de leur activité. Cet ensemble est à relier aux diverses formes d'interventions didactiques et aux ressources que les élèves mobilisent en réaction aux propositions des enseignants ou aux différents débats entre pairs. II est également nécessaire de poursuivre le travail de conception, d'expérimentation et de mise à disposition de contenus adaptés à ces activités par les enseignants. "D'ailleurs les contributions relatives aux séquences didactiques montrent en outre qu'au-delà des 
eJRIEPS Hors série n¹ Décembre 2015

aspects proprement méthodologiques, ce type d'intervention conduit à d'importantes transformations des attitudes pédagogiques et constitue en soi un excellent moyen de formation des enseignants » (Bronckart, 2004, p. 122).

Une bonne façon de faire « d'une pierre deux coups ».

\section{Bibliographie}

Austin, J.L. (1962). How to do things with words. Oxford : University Press.

Bride, J. (2015). Le karaté, patrimoine vivant immatériel. Médiation interculturelle des pratiques japonaises / françaises. Thèse de doctorat (non publiée) en Sciences du Sport. Université de Franche-Comté

Bronckart, J.P. (1996). Activité langagière, textes et discours. Pour un interactionnisme socio-discursif. Lausanne : Delachaux \& Niestlé.

Bronckart, J.-P. (2004). Commentaires conclusifs. Pour un développement collectif de l'interactionnisme socio-discursif . Calidoscópio. São Leopoldo : Universidade do Vale do Rio dos Sinos, no 2 (2), 113-123.

Brousseau, G. (1986). Fondements et méthodes de la didactique des mathématiques. Recherches en didactique des mathématiques, $7(2)$, 33-115.

Brousseau, G. (1998). Théorie des situations didactiques. Grenoble : La Pensée Sauvage.

Caverni, J.P. (1988) La verbalisation comme source d'observables du fonctionnement cognitif. In J.P. Caverni, C. Bastien, P. Mendelsohn \& G. Tiberghien, G. Psychologie cognitive : modèles et méthodes (pp. 253-273). Grenoble : Presses Universitaires.

Chang, C. W. (2006). Vers une approche constructiviste de l'enseignement du basket-ball à Taïwan. Thèse (non publiée). National Taïwan Normal University.

Christina, R.W. (1987). Motor learning: Future lines of research. In M.J. Safrit \& H.M. Eckert (Eds.), The cutting edge in physical education and exercise science research (American Academy of Physical Education Papers No. 20, pp. 26-41). Champaign, IL: Human Kinetics.

Clot, Y. (2000). Analyse psychologique du travail et singularité de l'action. In J.-M. Barbier (Ed.). L'analyse de la singularité de l'action, séminaire du CRF CNAM. Paris : Presses Universitaires de France.

Darnis, F. (2004). Place et rôle du développement cognitif et de l'interaction sociale dans l'acte tactique en jeux et sports collectifs. Thèse (non publiée). Université Victor Ségalen Bordeaux 2.

Darnis, F., Lafont, L., Menaut, A. (2007). Interactions verbales en situation de co-construction de règles d'action au hand-ball : L'exemple de deux dyades à fonctionnement contrasté. e Journal de la Recherche sur l'Intervention en Education Physique et sportive, 11, 56-17.

Deleplace, R. (1979). Rugby de mouvement, rugby total. Paris : Revue EPS.

Deriaz, D., Poussin, B., \& Gréhaigne, J.-F. (1998). Le débat d'idées. Éducation physique et Sport, 273, 80 82.

Dietsch, G. (2014). Le modèle du « futsal » en EPS : une forme de pratique scolaire du football en milieu difficile. Mémoire de mastère (non publié). Université Paris-Est Créteil.

Dietsch, G. (2015). Approche technologique et forme de pratique scolaire du football en milieu difficile. $e$ Journal de la Recherche sur l'Intervention en Education Physique et sportive, 35, 60-85.

Gréhaigne, J.-F. (1992). L'organisation du jeu en football. Paris : ACTIO. (Collection Actualité, Recherche, Sport dirigée par Michel Laurent et Pierre Therme).

Gréhaigne, J.-F., \& Deriaz, D. (2007). Le débat d'idées. in J. F. Gréhaigne (Coord.), Configurations du jeu, débat d'idées \& apprentissage du football et des sports collectifs (p. 111-119). Besançon: Presses universitaires de Franche-Comté.

Gréhaigne, J.-F., \& Godbout, P. (1998). Observation, critical thinking and transformation: Three key elements for a constructivist perspective of the learning process in team sport. In R. S. Feingold, C. R. Rees, G. T. Barrette, L. Fiorentino, S. Virgilio, \& E. Kowalski (Eds.), Education for life (pp. 109-118). Garden City (NY): Adelphi University.

Gréhaigne, J.F., \& Laroche, J.-Y. (1994) Quelques fondements et présupposés théoriques d'une démarche. In Groupe sports collectifs de l'Académie de Dijon. (ed.) Didactique des sports collectifs à l'école (pp 12-15) ? Paris : Dossiers E.P.S 17.

Gréhaigne, J.-F., Marle, P., \& Zerai, Z. (2013). Modèles, analyse qualitative et configurations prototypiques dans les sports collectifs. e Journal de la Recherche sur l'Intervention en Education Physique et sportive, 30, 05-25 


\section{eJRIEPS Hors série n¹ Décembre 2015}

Jacques, F. (1979). Dialogiques. Recherches logiques sur le dialogue. Paris: Presses Universitaires de France.

Joshua, S., \& Dupin, J.J. (1993). Introduction à la didactique des sciences et des mathématiques. Paris : Presses Universitaires de France.

Lafont, I., \& Winykamen, F. (1999). Co-operation and competition in children and adolescents. In Y. van den Auweele, F. Bakker, S. Biddle, M. Durand, R. Seiler (Eds.) Psychology for physical educators (pp. 379-404). Champaign, IL. : Human Kinetics.

Lave, J., \& Wenger, E. (1991). Situated learning : legitimate peripheral participation. New York : Cambridge University Press.

Le Bas. A (2008). Situation de pratique scolaire : transposition didactique et problématisation. Présentation orale au Colloque "Les didactiques et leurs rapports à l'enseignement et à la formation. Quel statut épistémologique de leurs modèles et de leurs résultats ?» Bordeaux 18-20 septembre 2008. IUFM d'Aquitaine.

Lesnes, M. (1977). Travail pédagogique et formation d'adulte : éléments d'analyse. Paris : Presses Universitaires de France.

Martinand, J.L. (1981). Pratiques sociales de référence et compétences techniques. A propos d'un projet d'initiation aux techniques de fabrication mécanique en classe de quatrième. In A. Giordan (Ed.) Diffusion et appropriation du savoir scientifique : enseignement et vulgarisation (pp. 227-249) Actes des troisièmes journées Internationales sur l'Éducation Scientifique, Université Paris 7.

Nachon, M. (2004). Interactions en Education Physique et Sportive : Le cas du Basket-ball. Approche des compétences sémiolangagières et construction de savoirs. Thèse (non publiée) en Science du langage, didactique, sémiotique. Université de Franche-Comté

Ochanine, D. (1978). Le rôle des images opératives dans la régulation des activités de travail. Psychologie et Education, 2, 63-72.

Ochanine, D., Quaas, W., \& Zaltzman, A. (1972). Déformation fonctionnelle des images opératives. Questions de Psychologie, 3.

Pastré, P. (1999). La conceptualisation dans l'action, bilan et nouvelles perspectives. Education permanente, 139, 13-35.

Perret-Clermont, A.N. (1979) La construction de l'intelligence dans l'interaction sociale. Bern : Peter Lang.

Piaget, J. (1974). Réussir et comprendre. Paris : PUF.

Poggi, M.-P., Musard, M., \& Wallian, N. (2007). Approche sociologique des interlocutions en EPS : le cas du basket-ball. Communication orale présentée au colloque international du Pôle Nord-Est des IUFM "Les effets des pratiques enseignantes sur les apprentissages des élèves ». Université de Besançon.

Poussin, B., \& Cotting, T. (2011). Effet d'une démarche didactique sur les prises de décision technicotactique en football. Bulletin de l'Association des Chercheurs Francophone en Football (ACFF), 6 , 18-22. http://blog.crdp-versailles.fr/acff/index.php/

Rabatel, A. (2004). Interactions orales en contexte didactique. Lyon : PUL.

Ricoeur, P. (1986). Du texte à l'action. Essais d'herméneutique II. Paris : Seuil.

Rochex, J.-Y. (2010). Approches cliniques et recherche en éducation. Questions théoriques et considérations sociales. Recherche \& Formation, 65, 111-122

Schubauer-Leoni, M.L. (1997). Interactions didactiques et interactions sociales : quels phénomènes et quelles constructions conceptuelles? Skholé, 7, 103-134.

Schön, D. (1994). Le praticien réflexif. À la recherche du savoir caché dans l'agir professionnel. Montréal : Éditions Logiques.

Sensevy, G. (1998). Institutions didactiques : étude et autonomie à l'école élémentaire. Paris : Presses Universitaires de France.

Sensevy, G. (2007) Des catégories pour décrire et comprendre l'action didactique. In G. Sensevy, A. Mercier, M.-L. Schubauer-Leoni, \& F. Leutenegger, (Eds.) Agir Ensemble : L'action didactique conjointe du professeur et des élèves (pp. 13-49). Rennes : Presses Universitaires de Rennes.

Sensevy, G., Mercier, A., Schubauer-Leoni, M.L., \& Leutenegger, F. (2007). Agir ensemble : l'action didactique conjointe du professeur et des élèves. Rennes : PUR.

Wallian, N., \& Chang, C. W. (2007). Sémiotique de l'action motrice et des activités langagières : vers une épistémologie des savoirs co-construits en sports collectifs. In J.F. Gréhaigne (Ed.), Configurations du jeu, débat d'idées et apprentissage des sports collectifs (pp. 145-164). Besançon: Presses de l'Université de Franche-Comté.

Wallian, N. (2010). Pluri-sémioticité et pluri-sémiotricité en Education Physique et Sportive. In A. Rabatel (Ed). Dire, montrer, agir, apprendre : les reformulations plurisémiotiques (pp. 217-236). Besançon : Presses universitaires de Franche-Comté. 
eJRIEPS Hors série n¹ Décembre 2015

Wallian, N. (2010 a). Sémiotique des interactions langagières entre pairs en éducation physique et sportive (EPS). Actes du Congrès international AREF «Actualités de la recherche en éducation et en formation ». Genève $(\mathrm{CH}), 13-16$ septembre 2010.

Zerai, Z. (2011). Apprentissage du handball chez les jeunes filles Tunisiennes et Françaises; apport de la verbalisation. Thèse de doctorat (non publiée) en Science du Sport. Université de FrancheComté.

Zerai, Z., Rezig, M., \& Zhigbi, M. (2008). Débats d'idées et apprentissage chez les filles en sport collectif. $e$ Journal de la Recherche sur l'Intervention en Education Physique et sportive, 13, 78-93. 